\title{
Dietary fibre and the pruritus of cholestatic jaundice
}

\author{
By M. A. EASTWOOD AND S. L. MOWBRAY \\ The University Department of Therapeutics, Royal Infirmary, Edinburgh \\ AND R. P. H. THOMPSON AND ROGER WILLIAMS \\ The Medical Research Council Group on Metabolism and Haemodynamics of Liver \\ Disease, Department of Medicine, and the Department of Chemical Pathology, \\ King's College Hospital, London \\ (Received I8 February 1970-Accepted 12 May 1970)

\begin{abstract}
I. In a double reversal experiment, diets containing either a normal or a large amount of lignin fibre were given to four patients with pruritus due to a liver disease.

2. Neither an improvement in symptoms nor a decrease in serum bile acid levels was found. The dose of fibre may have been insufficient, changes in intraluminal $\mathrm{pH}$ may have impaired adsorption of bile acids, or, less likely, the concentration of bile acids in the intestine may have been too low.
\end{abstract}

The concentrations of serum bile acids are frequently raised in patients with the pruritus of cholestatic jaundice (Haslewood, i 967). Although the concentrations in the skin may be more closely correlated with the intensity of pruritus (Schoenfield, Sjövall $\&$ Perman, 1967), measures which decrease bile acids in the serum often give relief. The resin cholestyramine binds bile acids within the lumen of the intestine and can relieve pruritus within a few days (Carey \& Williams, I96r). Another bile acidsequestrating agent is lignin, which is a phenyl propane polymer in vegetable fibre (Eastwood \& Hamilton, 1968). Since it strongly adsorbs bile acids, an increase of the fibre content of the diet might decrease the intestinal reabsorption of bile acids and relieve pruritus. In this paper we describe a double reversal experiment, in which the fibre content of the diets of four patients with chronic intrahepatic cholestasis and pruritus was either increased or unchanged. Pruritus was assessed from diaries kept by the patients and serial measurements were made of the serum levels of cholic, chenodeoxycholic and deoxycholic acids.

\section{EXPERIMENTAL}

\section{Patients studied}

Patient I. In this 44-year-old woman sarcoidosis had been diagnosed 2 years previously on the basis of enlarged hilar glands, a positive Kveim test, and sarcoid granulomata in lymph gland and liver tissue. She then developed jaundice, pruritus, pigmentation and hepatomegaly. Serum mitochondrial antibodies were present, and liver function tests were typical of primary biliary cirrhosis, with an increased level of IgM immunoglobulin. Cholestyramine improved the pruritus.

Patient 2. This 67 -year-old woman had a 2-year history of jaundice and pruritus 
with pigmentation and hepatosplenomegaly. Serum mitochondrial antibodies were absent. At laparotomy an established cirrhosis was found, the hepatic histological appearances being those of an active cirrhosis. Pruritus was controlled by cholestyramine.

Patient 3. A 73-year-old woman with jaundice and itching for 3 years had hepatosplenomegaly; laparotomy revealed a normal biliary tree. Histological appearances of a liver biopsy were compatible with primary biliary cirrhosis, and serum mitochondrial antibodies were demonstrated. Cholestyramine did not control itching.

Patient 4. A 46-year-old woman with a diagnosis of primary biliary cirrhosis had itched for 7 years, with hepatomegaly and raised serum alkaline phosphatase and bilirubin levels. Serum mitochondrial antibodies were present and a percutaneous transhepatic cholangiogram was negative. Cholestyramine partially controlled itching but its taste distressed the patient.

Patients I, 2 and 4 stopped taking cholestyramine 3 weeks before the trial, and patient 3 some months earlier.

\section{Design of trial}

Biscuits with either a negligible, or $28 \%$ by weight, fibre content were added to the patients' normal diets for four alternating periods of 2 weeks each. The diet in the first period was allocated at random. With the high-fibre biscuits the fibre content of the diet was increased by $14 \mathrm{~g} / \mathrm{d}$. The presentation of the biscuits was identical and they were of equal calorific value.

The patients were interviewed each week. Twice each day they recorded the severity of their pruritus during the previous $\mathrm{I} 2 \mathrm{~h}$, the frequency of bowel movements, and the occurrence of indigestion or of other side-effects. Weekly blood samples were taken for assessment of liver function and for measurement of bile acid concentrations before and during the trial period.

\section{Estimation of bile acids}

Serum was extracted with 5 vol. isopropanol, the extract dried and the residue dissolved in $2.5 \mathrm{~N}$-sodium hydroxide. Lipids were extracted before and after the hydrolysis of the conjugated bile acids, at $15 \mathrm{lb} / \mathrm{in}^{2}\left(\mathrm{I} \mathrm{kg} / \mathrm{cm}^{2}\right)$ at $110^{\circ}$ for $3 \mathrm{~h}$. The bile acids were extracted from the acidified hydrolysate with diethyl ether and separated into cholic, chenodeoxycholic and deoxycholic acids by thin-layer chromatography using silica gel cleaned of 'fines', iron and organic contaminants. Bile acids were identified using pyrene $(0.05 \%)$ in light petroleum (b.p. 60-80 ) (Eastwood \& Hamilton, 1967), and after its removal they were estimated by a modified Pettenkofer reaction (Boyd, Eastwood \& MacLean, 1966) at $42^{\circ}$. The recoveries for taurocholic, glycochenodeoxycholic and taurodeoxycholic acids $(5 \mu \mathrm{g})$ were $110 \pm 9 \%, 87 \pm 19 \%$ and $109 \pm 21 \%$ respectively (mean $\pm \mathrm{SD}$ ).

\section{RESULTS}

The patients found both types of biscuit palatable but preferred the control biscuit with a low fibre content. Neither biscuit affected bowel habit, nor was there any 
improvement in pruritus. Tests of liver function fluctuated, but the changes did not correlate with the fibre content of the diet. They are summarized in Table $\mathrm{I}$. The concentration of the three bile acids also fluctuated (Fig. I) independently of the dietary fibre and of the severity of itching. The greatest variations were found in the cholic acid concentrations, with values ranging from normal to eleven times normal. The levels of chenodeoxycholic acid ranged up to five times the normal, but those of deoxycholic acid rarely exceeded the normal value.

Table 1. Liver function tests in the four patients before and during the 2-week periods when they were given biscuits of normal and high fibre content

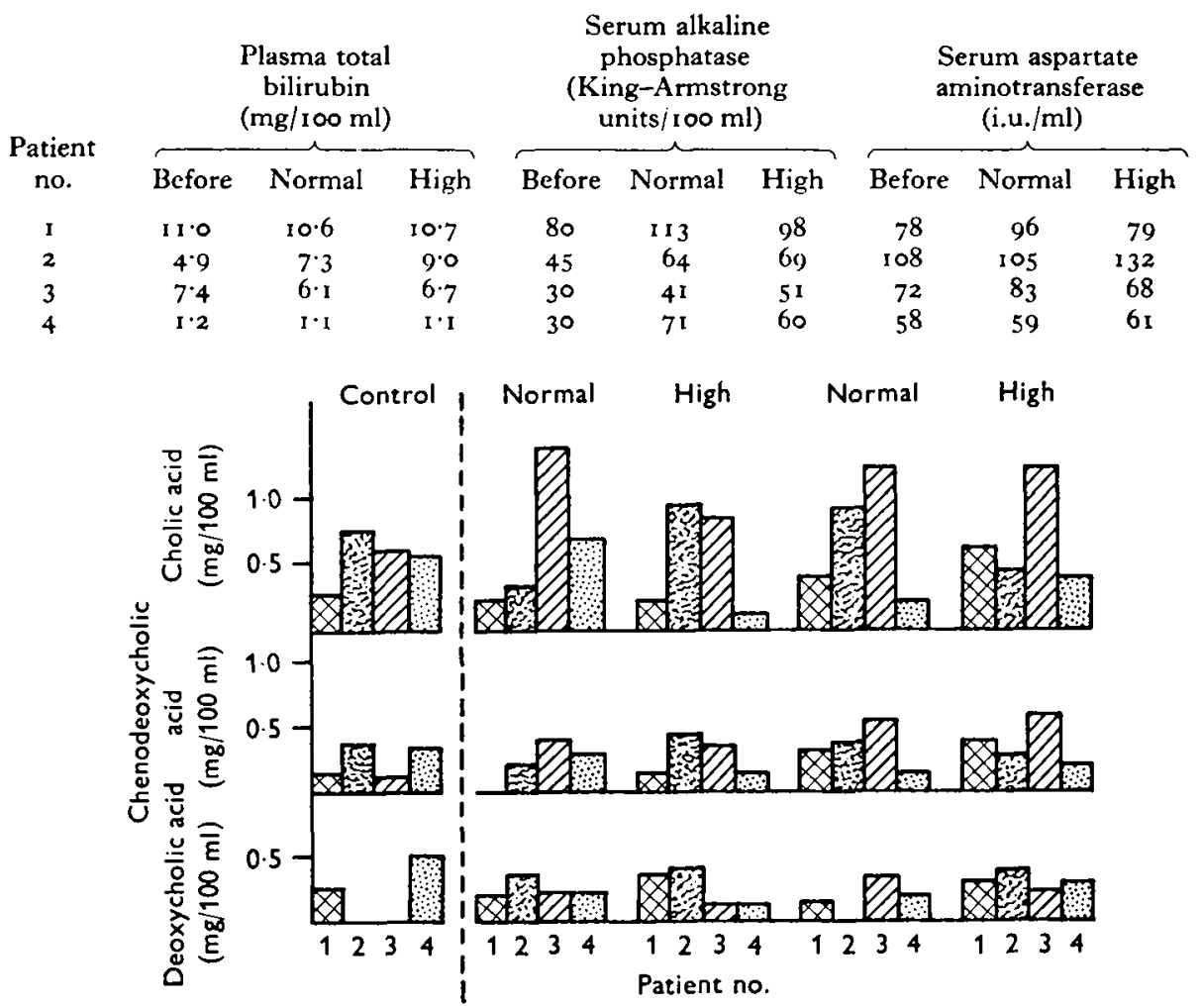

Fig. 1. Serum cholic, chenodeoxycholic, and deoxycholic acid levels in the four patients before and during the four, alternating 2 -week periods when they were given biscuits of either normal or high fibre content. Mean values for each period are given. For simplification, the sequence of biscuits is shown in the same order for all patients. Normal range for bile acids : $0.08-0.16 \mathrm{mg} /$ $100 \mathrm{ml}$ for cholic, $0.10-0.18$ for chenodeoxycholic and $0.12-0.29$ for deoxycholic acid.

\section{DISCUSSION}

The assessment of pruritus is difficult, but neither patients nor observers noticed any improvement during the trial. In addition, fibre was less effective than cholestyramine, for patient 2 obtained more relief from cholestyramine which she took both before and after the trial of fibre, and patients I and 4 preferred the cholestyramine but did not restart it because of its taste. 
The considerable variations in the levels of bile acids in the serum are surprising, especially as sometimes they fell to normal with no relief of pruritus. In general, high serum concentrations of the primary cholic and chenodeoxycholic acids, which are derived directly from cholesterol, were associated with low concentrations of the secondary deoxycholic acid. Deoxycholic acid is derived from cholic acid by the activity of caecal flora (Norman \& Shorb, 1962), which suggests that the increase of the serum bile acids is the result of reduced biliary excretion rather than of increased reabsorption. The results of the tests of liver function, the presence of deoxycholic acid in the serum, and the previous responses of the pruritus to cholestyramine all make it unlikely that in any patient biliary obstruction was so severe that levels of bile acids in the intestine were too low for fibre to be effective. Measurements of jejunal and faecal bile acids could have clarified this point.

The amount of fibre given may have been insufficient to bind significant quantities of bile acids, or the adsorption to the lignin in the bowel may have been too weak. Lignin adsorbs bile acids (Eastwood \& Hamilton, 1968), the amount of adsorption depending upon the degree to which its hydroxyl and carboxyl groups are ionized. Methylation of its acidic groups or a low $\mathrm{pH}$ decreases this ionization and increases adsorption so that, at the $\mathrm{pH}$ of the duodenum $(4 \cdot 7-6 \cdot 5)$, lignin will bind bile acids more strongly than at the $\mathrm{pH}$ of the ileum $(6 \cdot 0-7 \cdot 5)$. In addition, bile acids vary in their ability to be adsorbed by lignin; deoxycholic acid is adsorbed more strongly than chenodeoxycholic, while cholic acid is the most weakly adsorbed. The extent to which lignin is methylated, and the particular bile acids in the lumen of the small intestine, will therefore determine the adsorption. The degree of methylation of the fibre is not known, and it is possible that bile acids bound in the duodenum may be later released in the more alkaline ileum or colon. Methylation of lignin would result in a more powerful bile acid-sequestrating agent resisting the effect of $\mathrm{pH}$.

We are indebted to Mr G. R. Davenport for his help, and to Miss Rhoda Anderson for technical assistance. The biscuits were the gift of the Energen Foods Co. Ltd. The work was supported by the Medical Research Council and the Wellcome Trust.

\section{REFERENCES}

Boyd, G. S., Eastwood, M. A. \& MacLean, N. (1966). J. Lipid Res. 7, 83. Carey, J. B. \& Williams, G. (1961). F. Am. med. Ass. 176, 432. Eastwood, M. A. \& Hamilton, D. (1967). Biochem. F. 105, 37 C. Eastwood, M. A. \& Hamilton, D. (1968). Biochim. biophys. Acta 152, 165. Haslewood, G. A. D. (1967). Bile Salts p. 64. London: Methuen.

Norman, A. \& Shorb, M. S. (1962). Proc. Soc. exp. Biol. Med. 1 ro, 552.

Schoenfield, I. J., Sjövall, J. \& Perman, E. (1967). Nature, Lond. 213, 93. 